\title{
AN INVARIANT OF DIFFERENCE FIELD EXTENSIONS
}

\section{RICHARD M. COHN ${ }^{1}$}

Introduction. Let the difference field ${ }^{2} \mathfrak{F}$ have the transformally algebraic extension ${ }^{3}(\$)$. We have previously defined ${ }^{4}$ two numerical invariants, order and effective order, of such an extension. In this note we introduce a new numerical invariant, the limit degree of the extension $(\mathbb{S}$ of $\mathfrak{F}$, which will be denoted by l.d. (\$F/ $\mathfrak{F})$.

We suppose, first, that $\mathbb{B}$ is finitely generated; say $\mathbb{B}=\mathfrak{F}\left\langle\alpha_{1}, \cdots\right.$, $\left.\alpha_{r}\right\rangle$. Let $S_{k}$ denote the set of the $\alpha_{i}$ and their first $k$ transforms. We denote by $d_{k}$ the degree of $F\left(S_{k+1}\right)$ with respect to $F\left(S_{k}\right), k=0,1$, $2, \cdots$. The $d_{k}$ form a nonincreasing sequence whose terms are finite integers for sufficiently large $k$. We define l.d. (\$)/F) to be the least value assumed by the $d_{k}$. It will be proved in $\S 1$ that the limit degree is independent of the choice of the set of generators $\alpha_{i}$.

If $(B)$ is any-not necessarily finitely-generated - transformally algebraic extension of $\mathfrak{F}$, we define l.d. (\$)/F) to be the maximum of the limit degrees of all finitely-generated sub-extensions of $\mathfrak{F}$, if this maximum exists, or $\infty$, if it does not. That this definition is consistent with the preceding follows from Theorem I below when it is restricted to finitely generated extensions.

If $\mathfrak{M}$ is an irreducible manifold of dimension zero over a difference field $\mathfrak{F}$ and $\Sigma$ the corresponding prime difference ideal, we define the

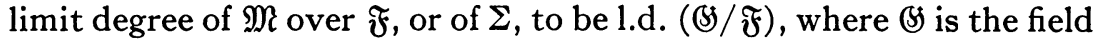
obtained by adjoining a generic zero of $\Sigma$ to $\mathfrak{F}$.

If $B$ is not transformally algebraic over $F$ the definition of limit degree may be applied and will always result in the value $\infty$. In this case it is more useful to consider the limit degree relative to a given basis of transformal transcendency. If $\Omega$ is the field formed by adjoining this basis to $\mathfrak{F}$, then 1.d. ((F/F) relative to the given basis is

Received by the editors June 17, 1955 .

${ }^{1}$ This investigation was supported in part by a grant from the Rutgers University Research Council.

${ }^{2}$ We refer the reader to [2] and [4] for the definition of basic concepts and the notations used in this paper.

$3[1$, p. 135].

$4[1$, p. 162]. The quantities $r$ and $s$ defined there are described as the order and effective order of a reflexive prime difference ideal. But they are defined in the first instance in terms of the properties of a certain field extension and we may also consider them to be the order and effective order of that extension. It is evident that the same procedure may be used in general to define the order and effective order of any extension. 
defined to be 1.d. $(\$ / \Omega)$. We define similarly the limit degree of a reflexive prime difference ideal or its irreducible manifold relative to a given set of parametric indeterminates.

We shall prove in $\$ 2$ the following fundamental result concerning limit degrees.

TheOREM I. Let $\mathfrak{G}$ be an extension of the difference field $\mathfrak{F}$, and let (5) be a difference field such that $\mathfrak{F} \subseteq \mathfrak{S} \subseteq \mathfrak{W}$. Then l.d. $(\mathfrak{S} / \mathfrak{F})$ is the

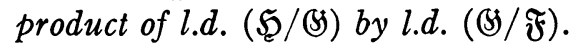

The following result, which permits the limit degree of a reflexive prime difference ideal to be determined from its characteristic set, will be shown to follow easily from Theorem I.

THEOREM II. Let $\Sigma$ be a reflexive prime difference ideal in $\mathfrak{F}\left\{u_{1}, \ldots\right.$, $\left.u_{q} ; y_{1}, \cdots, y_{p}\right\}$, the $u_{i}$ forming a parametric set. Let $A_{10}, \cdots, A_{1 r_{1}}$; $A_{20}, \cdots, A_{2 r_{2}} ; \cdots ; A_{p 0}, \cdots, A_{p r_{p}}$ be a characteristic set for $\Sigma$ with $A_{i 0}$ introducing $y_{i}, i=1, \cdots, p$. Let $d_{i}, i=1, \cdots, p$, denote the degree of $A_{i r_{i}}$ in the highest transform of $y_{i}$ which it contains. Then the limit degree of $\Sigma$ relative to the $u_{i}$ is $d_{1} \cdots d_{p}$.

1. Proof of invariance. We suppose that $(B)$ is a finitely-generated transformally algebraic extension of the difference field $\mathfrak{F}$; and that $\mathfrak{B}=\mathfrak{F}\left\langle\alpha_{1}, \cdots, \alpha_{r}\right\rangle=F\left\langle\beta_{1}, \cdots, \beta_{s}\right\rangle$. For $k=0,1,2, \cdots$, we denote by $S_{k}$ the set of the $\alpha_{i}, i=1, \cdots, r$, and their first $k$ transforms; by $T_{k}$ the set of the $\beta_{i}, i=1, \cdots, s$, and their first $k$ transforms.

There is a positive integer $h$ such that $\mathfrak{F}\left(S_{h}\right) \supseteq \mathfrak{F}\left(T_{0}\right)$ and $\mathfrak{F}\left(T_{h}\right)$ $\supseteq \mathfrak{F}\left(S_{0}\right)$. Then, for every positive integer $m, \mathfrak{F}\left(S_{h+m}\right) \supseteq \mathfrak{F}\left(T_{m}\right)$ and $\mathfrak{F}\left(T_{h+m}\right) \supseteq \mathfrak{F}\left(S_{m}\right)$.

Let $d_{1}$ denote the limit degree of $(S)$ with respect to $\mathfrak{F}$ as computed from the $\alpha_{i}$, and let $d_{2}$ be the limit degree computed using the $\beta_{i}$. Then for large $m$ and all positive integers $n \geqq h, d_{1}^{n+h}$ is the degree of $\mathfrak{F}\left(S_{m+n+h}\right)$ with respect to $\mathfrak{F}\left(S_{m}\right)$. But $\mathfrak{F}\left(S_{m+n+h}\right) \supseteq \mathfrak{F}\left(T_{m+n}\right)$ while $\mathfrak{F}\left(S_{m}\right) \subseteq \mathfrak{F}\left(T_{m+h}\right)$ so that this degree is not less than the degree of $\mathfrak{F}\left(T_{m+n}\right)$ with respect to $\mathfrak{F}\left(T_{m+h}\right)$. For large $m$ the latter is $d_{2}^{n-h}$. Hence, for all $n \geqq h$,

$$
d_{1}^{n+h} \geqq d_{2}^{n-h} .
$$

We may prove similarly that

$$
d_{2}^{n+h} \geqq d_{1}^{n-h}, \quad n \geqq h .
$$

It readily follows that $d_{1}=d_{2}$. This completes the proof of invariance.

2. Proof of Theorem I. We use the notation of Theorem I. We as- 
sume first that $\mathfrak{W}$ is a finitely generated extension of $\mathfrak{F}$. Then, by the Theorem of [3], $B$ is finitely generated. Suppose $\mathbb{S}=\mathfrak{F}\left\langle\alpha_{1}, \cdots, \alpha_{r}\right\rangle$, $\mathfrak{S}=\mathscr{S}\left\langle\beta_{1}, \cdots, \beta_{s}\right\rangle$. We denote by $S_{k}, k=0,1,2, \cdots$, the set consisting of the $\alpha_{i}$ and their first $k$ transforms, by $T_{k}, k=0,1,2, \cdots$, the set of the $\beta_{i}$ and their first $k$ transforms. We need consider only the case that $H$ is transformally algebraic over $F$.

We choose a sufficiently large fixed integer $t$. The meaning of "sufficiently large" will be clear from what follows. Defining

$$
d=1 . \mathrm{d} .(\mathfrak{W} / \mathfrak{F}), \quad d_{1}=1 . \mathrm{d} .(\mathfrak{\$} / \mathfrak{F}), \quad d_{2}=1 . \mathrm{d} . \quad(\$ / \mathfrak{S}),
$$

we find

(a) For $h=0,1,2, \ldots$ the degree of $\mathfrak{F}\left(S_{t+h}, T_{t+h}\right)$ with respect to $\mathfrak{F}\left(S_{t}, T_{t}\right)$ is $d^{h}$.

The degree of $\mathfrak{F}\left(S_{t+h}\right)$ with respect to $\mathfrak{F}\left(S_{t}\right)$ is $d_{1}^{h}$, so that the degree $n$ of $\mathfrak{F}\left(S_{t+h}, T_{t}\right)$ with respect to $\mathfrak{F}\left(S_{t}, T_{t}\right)$ is at most $d_{1}^{h}$. To obtain an opposite inequality we select a set $V$ which is a transcendency basis of $\mathfrak{F}\left(S_{t}, T_{t}\right)$ with respect to $\mathfrak{F}\left(S_{t}\right)$. Let $p$ denote the degree of $\mathfrak{F}\left(S_{t}, T_{t}\right)$ with respect to $\mathfrak{F}\left(S_{t}, V\right)$. Since $\mathfrak{F}\left(S_{t+h}\right)$ is algebraic over $\mathfrak{F}\left(S_{t}\right)$ no nonzero polynomial with coefficients in $\mathfrak{F}\left(S_{t+h}\right)$ is annulled by elements of $V$. Hence, the degree of $\mathfrak{F}\left(S_{t+h}, V\right)$ with respect to $\mathfrak{F}\left(S_{t}, V\right)$ is $d_{1}^{h}$. From the inclusion relations

$$
\begin{aligned}
& \mathfrak{F}\left(S_{t}, V\right) \subseteq \mathfrak{F}\left(S_{t}, T_{t}\right) \subseteq \mathfrak{F}\left(S_{t+h}, T_{t}\right), \\
& \mathfrak{F}\left(S_{t}, V\right) \subseteq \mathfrak{F}\left(S_{t+h}, V\right) \subseteq \mathfrak{F}\left(S_{t+h}, T_{t}\right)
\end{aligned}
$$

we find $p n=d_{1}^{h} m$, where $m$ is the degree of $\mathfrak{F}\left(S_{t+h}, T_{t}\right)$ with respect to $\mathfrak{F}\left(S_{t+h}, V\right)$. Hence $n \geqq d_{1}^{h} / p$.

Combining this with our previous inequality $n \leqq d_{1}^{h}$, we have

(b) The degree of $\mathfrak{F}\left(S_{t+h}, T_{t}\right)$ with respect to $\mathfrak{F}\left(S_{t}, T_{t}\right), h=0,1$, $2, \cdots$, is not less than $d_{1}^{h} / p$ and not more than $d_{1}^{h}$.

The degree of $\$\left(T_{t+h}\right)$ with respect to $\$\left(T_{t}\right)$ is $d_{2}^{h}$. Hence the degree of $\mathfrak{F}\left(S_{t+h}, T_{t+h}\right)$ with respect to $\mathfrak{F}\left(S_{t+h}, T_{t}\right)$ is at least $d_{2}^{h}$. We obtain an opposite inequality by the following considerations.

For $k$ sufficiently large the degree of $\mathfrak{F}\left(S_{t+k}, T_{t+1}\right)$ with respect to $\mathfrak{F}\left(S_{t+k}, T_{t}\right)$ is the degree of $\mathbb{S}\left(T_{t+1}\right)$ with respect to $\mathfrak{S}\left(T_{t}\right)$, or $d_{2}$. It follows that the degree of $\mathfrak{F}\left(S_{t+a}, T_{t+b+1}\right)$ with respect to $\mathfrak{F}\left(S_{t+a}\right.$, $\left.T_{t+b}\right), a-b \geqq k$, is at most $d_{2}$, since the $(t+b+1)$ th transforms of the $\beta_{i}$ satisfy equations over $\mathfrak{F}\left(S_{t+a}, T_{t+b}\right)$ which are the $b$ th transforms of those satisfied over $\mathfrak{F}\left(S_{t+k}, T_{t}\right)$ by the $(t+1)$ th transforms of the $\beta_{i}$.

On the other hand, if we denote the degree of $\mathfrak{F}\left(S_{t}, T_{t+1}\right)$ with respect to $\mathfrak{F}\left(S_{t}, T_{t}\right)$ by $d_{3}$, then, for any $a \geqq b \geqq t$ the degree of $\mathfrak{F}\left(S_{a}, T_{b+1}\right)$ with respect to $\mathfrak{F}\left(S_{a}, T_{b}\right)$ is at most $d_{3}$. 
We consider $h>k$. Then the degree of $\mathfrak{F}\left(S_{t+h}, T_{t+h}\right)$ with respect to $\mathfrak{F}\left(S_{t+h}, T_{t}\right)$ is the product of the degree of $\mathfrak{F}\left(S_{t+h}, T_{t+h}\right)$ with respect to $\mathfrak{F}\left(S_{t+h}, T_{t+h-k}\right)$ by the degree of the latter field with respect to $\mathfrak{F}\left(S_{t+h}, T_{t}\right)$. By the above the product is at most $d_{2}^{h-\boldsymbol{k}} d_{3}^{\mathbf{k}}=d_{2}^{h} q$, where $q=d_{3}^{k} / d_{2}^{k}$. We have thus proved

(c) The degree of $\mathfrak{F}\left(S_{t+h}, T_{t+h}\right)$ with respect to $\mathfrak{F}\left(S_{t+h}, T_{t}\right)$, $h=0,1,2, \cdots$, is not less than $d_{2}^{h}$ and not more than $d_{2}^{h} q$. (The case $h<k$ is unimportant, but the statement is obviously valid then also, though not proved above.)

Combining (a), (b), and (c) we have the inequality $(1 / p)\left(d_{1}^{h} d_{2}^{h}\right)$ $\leqq d^{h} \leqq d_{1}^{h} d_{2}^{h} q, h=0,1,2, \cdots$. By letting $h \rightarrow \infty$ we prove Theorem I for the case of finitely generated extensions $\mathfrak{S}$.

We now consider the case that $\mathfrak{W}$ is not necessarily finitely generated. Defining $d, d_{1}, d_{2}$ as before we note that if either $d_{1}$ or $d_{2}=\infty$, then $d=\infty$. Suppose $d_{1}$ and $d_{2}$ are both finite. Then there is a set $\alpha_{1}, \cdots, \alpha_{r}$ in (S) such that 1.d. $\left(\mathfrak{F}\left\langle\alpha_{1}, \cdots, \alpha_{r}\right\rangle / \mathfrak{F}\right)=d_{1}$, and also a

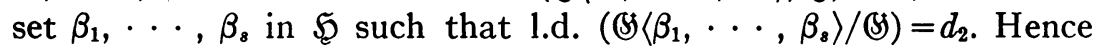
1.d. $\left(\mathfrak{F}\left\langle\alpha_{1}, \cdots, \alpha_{r} ; \beta_{1}, \cdots, \beta_{s}\right\rangle / \mathfrak{F}\left\langle\alpha_{1}, \cdots, \alpha_{r}\right\rangle\right) \geqq d_{2}$. By the preceding case of Theorem I, 1.d. $\left(\mathfrak{F}\left\langle\alpha_{1}, \cdots, \alpha_{r} ; \beta_{1}, \cdots, \beta_{s}\right\rangle / \mathfrak{F}\right) \geqq d_{1} d_{2}$. Hence $d \geqq d_{1} d_{2}$. Suppose $d>d_{1} d_{2}$. Then there is a set $\gamma_{1}, \cdots, \gamma_{t}$ in $\mathfrak{S}$ such that 1.d. $\left(\mathfrak{F}\left\langle\gamma_{1}, \cdots, \gamma_{t}\right\rangle / \mathfrak{F}\right)>d_{1} d_{2}$. Then for any set $\delta_{1}, \cdots, \delta_{p}$ in (अ) 1.d. $\left(\mathfrak{F}\left\langle\gamma_{1}, \cdots, \gamma_{t} ; \delta_{1}, \cdots, \delta_{p}\right\rangle / \mathfrak{F}\right)>d_{1} d_{2}$. But 1.d. $\left(\mathfrak{F}\left\langle\gamma_{1}, \cdots, \gamma_{t}\right.\right.$; $\left.\left.\delta_{1}, \cdots, \delta_{p}\right\rangle / \mathfrak{F}\right)$ is the product of

$$
\text { l.d. }\left(\mathfrak{F}\left\langle\gamma_{1}, \cdots, \gamma_{t} ; \delta_{1}, \cdots, \delta_{p}\right\rangle / \mathfrak{F}\left\langle\delta_{11}, \cdots, \delta_{p}\right\rangle\right)
$$

and 1.d. $\left(\mathfrak{F}\left\langle\delta_{1}, \cdots, \delta_{p}\right\rangle / \mathfrak{F}\right)$, and the latter factor is at most $d_{1}$. It follows that 1.d. $\left.\left(\mathfrak{F}\left\langle\gamma_{1}, \cdots, \gamma_{t} ; \delta_{1}, \cdots, \delta_{p}\right\rangle / \mathfrak{F}\left\langle\delta_{1}, \cdots, \delta_{p}\right\rangle\right)\right\rangle d_{2}$ for all sets $\delta_{1}, \cdots, \delta_{p}$. But 1.d. $\left(\mathfrak{S}\left\langle\gamma_{1}, \cdots, \gamma_{t}\right\rangle / \mathfrak{S}\right)=d_{3} \leqq d_{2}$, whence it follows readily that, for some finite set $\delta_{1}, \cdots, \delta_{p}$, l.d. $\left(\mathfrak{F}\left\langle\gamma_{1}, \cdots, \gamma_{t}\right.\right.$; $\left.\left.\delta_{1}, \cdots, \delta_{p}\right\rangle / \mathfrak{F}\left\langle\delta_{1}, \cdots, \delta_{p}\right\rangle\right)=d_{3}$. For there is some integer $h$ such that we may find an equation for $\gamma_{1 h}$ with coefficients in the ring $\mathbb{S}_{1}=\mathbb{S}\left[\gamma_{1}, \cdots, \gamma_{1, h-1} ; \gamma_{2}, \cdots, \gamma_{2, h-1} ; \cdots ; \gamma_{t}, \cdots, \gamma_{t, h-1}\right]$, for $\gamma_{2 h}$ in $\mathfrak{S}_{1}\left[\gamma_{1 h}\right]$, for $\gamma_{3 h}$ in $\mathfrak{S}_{1}\left[\gamma_{1 h}, \gamma_{2 h}\right]$, and so on, such that the product of the degrees of these equations is $d_{3}$. We choose the set $\delta_{1}, \cdots, \delta_{p}$ to consist of the coefficients of the products of the $\gamma_{i j}$ in these equations. We have thus obtained a contradiction. This completes the proof of Theorem I.

3. Computation of the limit degree. The limit degree of a reflexive prime difference ideal $\Sigma$ in $\mathfrak{F}\{y\}$ is evidently the degree of the highest polynomial of its characteristic set in the highest transform of $y$ which it effectively involves. From this fact and Theorem IX of [1] 
we immediately obtain Theorem II. Another immediate consequence is

TheoRem III. Let $A$ be an algebraically irreducible difference polynomial in $\mathfrak{F}\left\{u_{1}, \cdots, u_{q} ; y\right\}$ of order and effective order $r$ in $y$ and degree $n$ in $y_{r}$. Then the sum of the limit degrees relative to the $u_{i}$ of the components of its general solution is $n$.

The proof follows immediately by Theorem VII of [1] when one takes into account the form of the characteristic sequences of $A$ as explained in the proof of Theorems IV and IV' of that paper.

With regard to the relations among the limit degrees of these components, we have at present, besides Theorem III, only the following very special result.

THEOREM IV. Let $\mathfrak{F}$ be an inversive difference field and $A$ an algebraically irreducible zero-order difference polynomial in $\mathfrak{F}\{y\}$ of degree $n$ in $y$. Then if any component of $A$ is of limit degree 1 there are $n$ components, each of limit degree 1.

The conclusion is not valid if the requirement that the field be inversive is omitted.

Proof of Theorem IV. To say that a zero-order extension $\mathfrak{F}\langle\gamma\rangle$ of $\mathfrak{F}$ is of limit degree 1 is equivalent to saying that it is of finite degree over $\mathfrak{F}$. Hence, there is a solution $\alpha$ of the difference equation $A=0$ such that $\mathfrak{F}\langle\alpha\rangle$ is of finite degree over $\mathfrak{F}$. Then, for some $r$, $\mathfrak{F}\langle\alpha\rangle=\mathfrak{F}\left(\alpha, \alpha_{1}, \cdots, \alpha_{h-1}\right)$.

Let $\alpha_{j}^{(i)}, j=0,1,2, \cdots ; i=1, \cdots, n$, with $\delta_{j}^{(1)}=\alpha_{j}$, be the $n$ solutions of the equation $A_{j}=0$ considered as an algebraic equation. To prove Theorem IV it is sufficient to show that the field $\&$ formed by adjoining the $\alpha_{j}^{(i)}, i=1, \cdots, n ; j=0,1,2, \cdots$, to $\mathfrak{F}$ is finite over $\mathfrak{F}$; for, if $\beta$ is any solution of the difference equation $A=0, \mathfrak{F}\langle\beta\rangle$ considered as a field, not as a difference field, is isomorphic to a subfield of $(\xi)$ and hence finite over $\mathfrak{F}$ if $\mathfrak{F}$ is finite over $\mathfrak{F}$. We shall in fact show that $\left(\mathcal{S}\right.$ is the field formed by adjoining to $\mathfrak{F}$ only those $\alpha_{j}^{(k)}$, $i=1, \cdots, n$, with $0 \leqq j<r$.

Let $s \geqq r$. Denote by $\beta_{s}$ some $\alpha_{s}^{(1)}, 1 \leqq i \leqq n$. We shall show that $\beta_{z}$ is contained in the field formed by adjoining to $\mathfrak{F}$ the $\alpha_{j}^{(t)}$, $i=1, \cdots, n$, with $j<s$. The last statement of the preceding paragraph, and hence Theorem IV, follow immediately from this.

If we map each element of $\mathfrak{F}$ onto its $(s-r)$ th transform we obtain, because $\mathfrak{F}$ is inversive, an automorphism of $\mathfrak{F}$ which carries the coefficients of $A$ into those of $A_{s-r}$. Hence this automorphism may 
be extended to an isomorphism of $\mathfrak{F}\left(\alpha_{r}\right)$ onto $\mathfrak{F}\left(\beta_{s}\right)$ with $\alpha_{r}$ mapping into $\beta_{s}$. It follows that $\mathfrak{F}\left(\alpha, \alpha_{1}, \cdots, \alpha_{r}\right)$ is isomorphic to an extension $\mathfrak{F}\left(\beta_{s-r}, \beta_{s-r+1}, \cdots, \beta_{s}\right)$ of $\mathfrak{F}\left(\beta_{s}\right)$. Evidently each $\beta_{j}, s-r \leqq j$ $\leqq s-1$, satisfies $A_{j}=0$, hence is an $\alpha_{j}^{()}$. Since $\alpha_{r}$ is a rational combination, with coefficients in $\mathfrak{F}$, of $\alpha, \cdots, \alpha_{r-1}$, it follows that $\beta_{\varepsilon}$ is a rational combination, with coefficients in $\mathfrak{F}$, of $\beta_{8-r}, \cdots, \beta_{s-1}$, and hence is in the field formed by adjoining to $\mathfrak{F}$ the $\alpha_{j}^{(i)}, j<s$. This completes the proof of Theorem IV.

\section{REFERENCES}

1. R. M. Cohn, Manifolds of difference polynomials, Trans. Amer. Math. Soc. vol. 64 (1948) pp. 133-172. 530.

2. —_, Extensions of difference fields, Amer. J. Math. vol. 74 (1952) pp. 507-

3. - Finitely generated extensions of difference fields, Proc. Amer. Math. Soc. vol. 6 (1955) pp. 3-5.

4. H. W. Raudenbush and J. F. Ritt, Ideal theory and algebraic difference equations, Trans. Amer. Math. Soc. vol. 46 (1939) pp. 445-452.

RUTGERS UNIVERSITY AND

INSTITUTE FOR ADVANCEd STUdy 